\title{
ARTICLE
}

Clinical Study

\section{Mouse-human co-clinical trials demonstrate superior anti-tumour effects of buparlisib (BKM120) and cetuximab combination in squamous cell carcinoma of head and neck}

\author{
Hye Ryun Kim ${ }^{1}$, Han Na Kang ${ }^{2}$, Mi Ran Yun ${ }^{2}$, Kwon Young Ju ${ }^{1}$, Jae Woo Choi ${ }^{3}$, Dong Min Jung ${ }^{3}$, Kyoung Ho Pyo ${ }^{2}$, Min Hee Hong ${ }^{1}$, \\ Myoung-Ju Ahn ${ }^{4}$, Jong-Mu Sun ${ }^{4}$, Han Sang Kim ${ }^{1}$, Jinna Kim ${ }^{5}$, Jinseon Yoo ${ }^{6}$, Kyu Ryung Kim ${ }^{6}$, Yoon Woo Koh ${ }^{7}$, Se Heon Kim ${ }^{7}$, \\ Eun Chang Choi ${ }^{7}$, Sun Ock Yoon ${ }^{8}$, Hyo Sup Shim (iD ${ }^{8}$, Soonmyung Paik ${ }^{3,9}$, Tae-Min Kim ${ }^{6}$ and Byoung Chul Cho (D) $^{1,2}$
}

\begin{abstract}
BACKGROUND: Recurrent and/or metastatic squamous cell carcinoma of head and neck (R/M SCCHN) is a common cancer with high recurrence and mortality. Current treatments have low response rates (RRs).

METHODS: Fifty-three patients with R/M SCCHN received continuous oral buparlisib. In parallel, patient-derived xenografts (PDXs) were established in mice to evaluate resistance mechanisms and efficacy of buparlisib/cetuximab combination. Baseline and ontreatment tumour genomes and transcriptomes were sequenced. Based on the integrated clinical and PDX data, 11 patients with progression under buparlisib monotherapy were treated with a combination of buparlisib and cetuximab.

RESULTS: For buparlisib monotherapy, disease control rate (DCR) was $49 \%$, RR was $3 \%$ and median progression-free survival (PFS) and overall survival (OS) were 63 and 143 days, respectively. For combination therapy, DCR was 91\%, RR was $18 \%$ and median PFS and OS were 111 and 206 days, respectively. Four PDX models were originated from patients enrolled in the current clinical trial. While buparlisib alone did not inhibit tumour growth, combination therapy achieved tumour inhibition in three of seven PDXs. Genes associated with apoptosis and cell-cycle arrest were expressed at higher levels with combination treatment than with buparlisib or cetuximab alone.

CONCLUSIONS: The buparlisib/cetuximab combination has significant promise as a treatment strategy for R/M SCCHN.

CLINICAL TRIAL REGISTRATION: NCT01527877.
\end{abstract}

British Journal of Cancer (2020) 123:1720-1729; https://doi.org/10.1038/s41416-020-01074-2

\section{BACKGROUND}

Squamous cell carcinoma of head and neck (SCCHN) is the sixth most frequent cancer with a dismal prognosis and high mortality. ${ }^{1}$ Low survival, in combination with the significant toxicity of current treatment strategies, emphasises the necessity for novel therapies. $^{2}$ In recurrent/metastatic SCCHN (R/M SCCHN), the only approved targeted therapy is cetuximab, a monoclonal antibody against the epidermal growth factor receptor (EGFR), with a response rate (RR) of $10-15 \%{ }^{3}$ Anti-programmed death 1 (PD-1) inhibitors, including pembrolizumab and nivolumab, were recently approved for SCCHN, which is refractory to platinumbased therapy. Although anti-PD-1 therapy showed improved outcomes over previous standard chemotherapies (taxane, methotrexate or cetuximab), objective responses have been reported in only $15-20 \%$ of patients. ${ }^{4-6}$
Recent genomic studies have suggested potential therapeutic opportunities. Genetic alterations of the phosphatidylinositol 3kinase (PI3K)-mTOR cell signalling pathway are common, particularly gain-of-function mutations of PI3K catalytic subunit $a$ and loss-of-function mutations of PTEN. ${ }^{7,8}$ Buparlisib (BKM120) is a novel, oral pan-PI3K inhibitor. Recently, buparlisib combined with paclitaxel showed improved efficacy in the treatment of $R / M$ SCCHN patients over paclitaxel alone, suggesting the importance of PI3K inhibition. ${ }^{9}$ We therefore conducted a Phase 2 study of buparlisib in R/M SCCHN.

To overcome the limited predictive value of conventional preclinical models, patient-derived tumour xenograft (PDX) models are a promising advance in oncology. ${ }^{10-12}$ These models, created by direct implantation of the patient's tumour into immunodeficient mice, preserve histologic and genetic

${ }^{1}$ Division of Medical Oncology, Yonsei Cancer Center, Yonsei University College of Medicine, Seoul, Korea; ${ }^{2} \mathrm{JE}-\mathrm{UK}$ Institute for Cancer Research, JEUK Co., Ltd, Gumi-City,

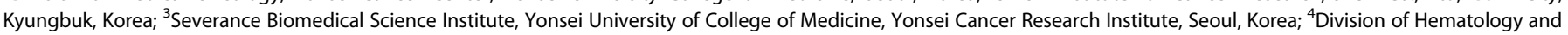

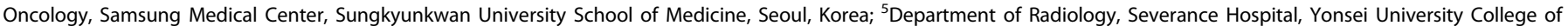

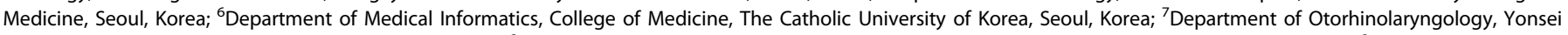

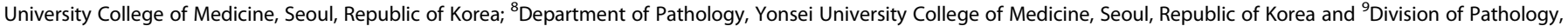
NSABP, Pittsburgh, PA, USA

Correspondence: Tae-Min Kim (tmkim@catholic.ac.kr) or Byoung Chul Cho (cbc1971@yuhs.ac)

These authors contributed equally: Hye Ryun Kim, Han Na Kang

These authors jointly supervised this work: Tae-Min Kim, Byoung Chul Cho

Received: 23 July 2019 Revised: 9 June 2020 Accepted: 2 September 2020

Published online: 23 September 2020 
characteristics of the original patient tumours. They have been shown to be predictive of clinical outcomes and are being used for translational research, preclinical drug screening and biomarker identification/validation. ${ }^{13-15}$ The mouse-human co-clinical trial is a new concept in which the treatment of interest is simultaneously tested in patients and PDX models derived from tumours of the patients enrolled in the clinical trial. ${ }^{15}$ This provides a platform to develop new combination treatments, identify predictive biomarkers and provide insights into resistance mechanisms.

We conducted a co-clinical trial mirroring an ongoing clinical study to identify predictive markers and optimal combinational strategies. We established PDX models that faithfully replicated the histologic, genomic and drug responses observed in the corresponding patients. We tested the efficacy of a combination of buparlisib and cetuximab on the PDX models. Based on the results, we revised the clinical trial protocol to treat patients with a combination of buparlisib and cetuximab, with promising results.

\section{METHODS}

Study design

This was a multicentre, Phase 2 study of buparlisib (100 mg/day) monotherapy in patients with R/M SCCHN who had progressed on platinum-based chemotherapy. The primary endpoint was disease control rate (DCR). Secondary endpoints included RR, progressionfree survival rate (PFS), overall survival (OS) and safety.

Patients with histologically confirmed R/M SCCHN were enrolled. Patients were at least 18 years old and had an Eastern Cooperative Oncology Group performance status (ECOG-PS) of $0-2$, at least one measurable disease and documented progressive disease after platinum-based chemotherapy for R/M SCCHN. Patients received continuous oral buparlisib ( $100 \mathrm{mg} /$ day) until disease progression, death or unacceptable adverse events (AEs). Treatment cycles were 28 days long. Drug doses were withheld and/or reduced for intolerable grade 2 or grade $3 / 4$ toxic effects. A maximum of two dose-level reductions was permitted $(80 \mathrm{mg}$, then $60 \mathrm{mg}$ ).

Response evaluations were defined according to RECIST 1.1 guidelines. ${ }^{16}$ Radiographic imaging was conducted at week 4, then at every 8 weeks thereafter until disease progression or when clinically indicated. Safety assessments included physical examinations, documentation of AEs and laboratory measurements on day 1 of each cycle. AEs were graded according to the Common Terminology Criteria for Adverse Events version 4.0. This study was conducted under approval by the institutional review boards of Severance Hospital and all patients provided informed consent.

We revised the treatment protocol to the combination for patients that progressed under buparlisib monotherapy. This revised protocol was approved by the institutional review boards of Severance Hospital. After protocol amendment, 11 patients were treated with buparlisib/cetuximab.

\section{PDX models}

A total of seven tumour samples were obtained from four patients (YHIM-01, -02, -06 and 07) with R/M SCCHN treated with buparlisib in the Phase 2 trial and from three patients (YHIM-03, -04 and 05) with SCCHN who had undergone surgery. Tumours and paired peripheral blood samples were collected prior to initial buparlisib treatment.

Six- to 8-week-old female severe combined immunodeficient (NOG) and nude (nu/nu) mice (OrientBio, Seoul, Korea) were used as recipients. After completion of experiments, we sacrificed mice by inhalation of anaesthetics with $\mathrm{CO}_{2}$. All mice models were maintained in the specific pathogen-free facility of the Avison BioMedical Research Center (ABMRC) Animal Research Center at Yonsei University College of Medicine. All methods were performed in accordance with the guidelines of the Animal Research Committee of Yonsei University College of Medicine and were approved by the Association of Assessment and Accreditation of Laboratory Animal Care. Patient tumour samples were cut into $\sim 3 \mathrm{~mm}$ cubes and implanted subcutaneously into six or seven mice for each patient. When tumours grew to $1.5 \mathrm{~cm}$ in diameter, they were excised, dissected into $\sim 3-\mathrm{mm}$ cubes and implanted into another set of mice by the same procedure. The passage harbouring the patient-derived material was termed F0, with subsequent generations numbered consecutively (F1, F2, etc.). The rest of the carcinoma was cryopreserved and processed for biological studies. Tumour cells from the third passage (F3) were expanded for the in vivo drug efficacy test.

\section{PDX-derived cell models}

F3 tumours were excised and chopped and/or sliced. Spill-out cells were also collected. Samples were incubated with collagenase/ dispase II for $1 \mathrm{~h}$ at $37^{\circ} \mathrm{C}$ in phosphate-buffered saline (PBS) and pelleted at 1500 r.p.m. for $10 \mathrm{~min}$. The cell pellet was gently resuspended in RPMI-1640 culture medium and cells were plated onto collagen-coated culture surfaces. Cultures were kept in a humidified $5 \% \mathrm{CO}_{2}$ atmosphere at $37^{\circ} \mathrm{C}$. On the following day, cultures were washed twice with $\mathrm{PBS}\left(37^{\circ} \mathrm{C}\right)$ to remove cell debris and nonadherent blood cells. Mouse Cell Depletion (MACS, 130104-694) and Cancer Isolation (MACS, 130-108-339) Kits were used to enrich for human tumour cells. The EpCAM-positive cell subpopulation in primary cultures was analysed using flow cytometry. Successfully established models were free of stromal cells and maintained a >95\% EpCAM-positive cell subpopulation. The medium was replenished with fresh medium every 3 days.

In vivo drug tests

When tumour volumes reached $200-250 \mathrm{~mm}^{3}$, mice were segregated into treatment groups based on tumour volume, growth rate and mouse weight. Vehicle ( $5 \mathrm{mM}$ citrate buffer) was administered to the control group. Ten mice per group were randomised and treated with vehicle or buparlisib $(35 \mathrm{mg} / \mathrm{kg}, \mathrm{QD}$, oral) alone or in combination with cetuximab $(10 \mathrm{mg} / \mathrm{kg}, \mathrm{Q} 3 \mathrm{D}$, intraperitoneally).

Tumour dimensions were measured twice a week with a digital calliper and tumour volume was calculated by the following formula: tumour volume $=\left[\right.$ length $\times$ width $\left.^{2}\right] / 2$. Percentage tumour growth inhibition $[\% \mathrm{TGI}=1-$ (change of tumour volume in treatment group/change of tumour volume in control group) $\times$ 100] was used for the evaluation of anti-tumour efficacy. ${ }^{15}$

Cell viability assays

Cells were seeded at 3000/well in 96-well clear-bottom microplates, incubated overnight and subsequently treated with drugs for 3 days. Cell viability was analysed using CellTiter-Glo according to the manufacturer's protocol. $I C_{50}$ values were calculated using v5.0 GraphPad Prism (GraphPad Software, Inc., La Jolla, CA, USA). Drugs used were cetuximab (Selleckchem A2000) and BKM120 (Selleckchem S2247). Combination indices (Cls) were calculated using the CacluSyn method. ${ }^{17}$ For colony formation assays, single cells were seeded onto 6-well plates at a density of 3000/well. After overnight attachment, cells were treated for 14 days. Medium including drugs was replaced every 3 days. After treatment, cells were washed with PBS, fixed in 4\% paraformaldehyde in PBS for $10 \mathrm{~min}$ and stained with $0.5 \%$ crystal violet in $20 \%$ methanol for $20 \mathrm{~min}$. To evaluate clonogenicity, images were captured using a flatbed scanner and cells were dissolved with $20 \%$ acetic acid in $20 \%$ methanol. The optical density of each well was read at $570 \mathrm{~nm}$ using a SpectraMax 250 microplate reader (Molecular Devices). ${ }^{18}$

Immunoblot analysis

Cell lysates containing equal amounts of protein were separated by sodium dodecyl sulfate-polyacrylamide gel electrophoresis and transferred to a membrane, and then probed with primary and 
1722

secondary antibodies. Signals were detected with SuperSignal ${ }^{\mathrm{TM}}$ West Pico Chemiluminescent Substrate (Thermo Fisher Scientific, MA, USA). Primary antibodies were against cyclin B (Cell Signaling Technology, cat. no. 122231), cyclin D (Cell Signaling Technology, cat. no. 8396), cyclin E (Santa Cruz Biotechnology, cat. no. 481), cleaved poly (ADP-ribose) polymerase (PARP) (Cell Signaling Technology, cat. no. 5625), cleaved caspase-3 (Cell Signaling Technology, cat. no. 9664), cleaved caspase-7 (Cell Signaling Technology, cat. no. 8438), BCL-2 (Cell Signaling Technology, cat. no. 2872), cleaved caspase-9 (Cell Signaling Technology, cat. no. 7237) and Myc (Cell Signaling Technology, cat. no. 9402). After washing, blots were probed with horseradish peroxidase-conjugated rabbit secondary antibodies (Cell Signaling Technology, Boston, MA, USA, cat. no. 7074). The membrane was stripped and re-probed with an anti- $\beta$-actin (Sigma-Aldrich, cat. no. A3854) antibody as an internal control.

\section{Histology}

Tissues from all PDX models were harvested and fixed in $10 \%$ buffered formalin within $30 \mathrm{~min}$ of resection, and then processed by routine procedures after $24 \mathrm{~h}$ fixation. Sections were stained with haematoxylin and eosin (H\&E) and reviewed by a pathologist to confirm SCCHN.

Whole-exome sequencing (WES) and somatic variants WES was used to identify somatic single-nucleotide variations or substitutions (SNVs) and short insertions/deletions (indels) by comparing the tumour genomes with matched normal genomes. To minimise false positives from xenocontamination, sequencing reads from host (mouse) genomes were filtered out from those from tumour (human) genomes using the Xenome software. ${ }^{19}$ Matched normal genomes were prepared from patients' peripheral blood. Exome-captured DNA by Agilent SureSelect Human All Exome $50 \mathrm{Mb}$ Kit (Agilent, USA) was used to prepare libraries and 100-bp paired-end sequencing was performed using an Illumina HiSeq2000 platform (Illumina, USA). Alignment of sequencing reads onto the human reference genome (hg19) was performed using BWA-MEM (Burrows-Wheeler aligner-mem). ${ }^{20}$ Local realignment and score recalibration of sequencing reads were performed using Genome Analysis ToolKit (GATK). ${ }^{21}$ SNVs and indels (somatic mutations, hereafter) were identified by MuTect and Indelocator, ${ }^{22}$ respectively, and collectively analysed as somatic mutations of PDTX tumours. Somatic copy number alterations (SCNAs) were identified by comparing the sequencing depth of tumour and matched normal genomes using Excavator. ${ }^{23}$

\section{Targeted deep-sequencing and germline variants}

Genomic DNA from formalin-fixed paraffin-embedded (FFPE) or fresh tissue, prepared using the QIAamp DNA FFPE Tissue Kit (Qiagen) or DNeasy Blood and Tissue Kit (Qiagen), was screened for 244 cancer-related genes using a customised SureSelect Kit (Agilent Technologies, Santa Clara, CA, USA) (Supplementary Table S1). Targeted sequencing was performed using an Illumina HiSeq2000 with an average coverage depth of $500-1000 \times$. The $\mathrm{BWA}^{20}$ with a hybrid human (hg19) and mouse (mm10) genome was used to separate 100 -bp paired-end sequence reads at PDX-F2 into human and mouse reads. Each read was aligned to both genomes and the best-matched read of the human genome was established. ${ }^{24}$ After pre-processing, the GATK ${ }^{21}$ was used for mark duplication, local realignment and base quality-score recalibration of the reads. The GATK Haplotype caller was run to identify germline mutations. Poorquality variants were filtered out using GATK VariantFiltration with the following criteria: read depth $<20$, quality by depth $<2.0$, Fisher strand $>60.0$, root-mean-square mapping quality $(\mathrm{MQ})<20.0$, MQRankSum $<-12.5$, ReadPosRankSum $<-8.0$ and mutant allele frequency (MAF) $<0.2$. ANNOVAR ${ }^{25}$ was used for variant annotation, and the $\mathrm{CIVIC}^{26}$ and DoCM ${ }^{27}$ databases were used to annotate mutations that could be used for targeted therapy.
RNA-sequencing-based gene expression profiling

To identify changes in gene expression, we used RNA-sequencing. A cDNA library was generated and $100 \mathrm{bp}$ paired-end sequencing was performed using Illumina HiSeq2000 (Illumina, USA). Spliceaware alignment was conducted using the TopHat aligner. ${ }^{28}$ Gene-level summaries of expression levels into fragments per kilobase million were performed using CuffLinks software. ${ }^{28}$

\section{Visualisation}

Oncoprint heatmaps were drawn for the patterns of overall mutations using the ComplexHeatmap. ${ }^{29}$ Lollipop plots were created for frequently mutated genes using MAFtools, ${ }^{30}$ which offers a multitude of analysis and visualisation modules that are commonly used in cancer genomic studies, to identify the recurrence of genomic loci with variants.

\section{Statistical analyses}

A Simon's two-stage design was used to test the null hypothesis (P0) with a $10 \%$ significance level that the objective response rate (ORR) is $\leq 30 \%$ vs. the alternative hypothesis (P1) that the ORR is $\geq 45 \%$. The expected sample size is 48 patients to provide $80 \%$ power to reject P0 when the true ORR is $45 \%$. Twenty-three will be accrued during stage $I$, and if six or fewer responses are observed in the first stage, the trial is stopped early. Allowing for a follow-up loss rate of $10 \%$, the total sample size is 53 patients. Based on the preclinical data, protocol amendment was in progress and at that time 42 patients were enrolled. After protocol amendment, additional 11 eligible patients were treated with buparlisib + cetuximab after failure to buparlisib.

Results are presented as the mean \pm standard deviation of at least three experiments for each group. Statistical differences were determined using analysis of variance and the Wilcoxon's ranksum test for independent samples. Kaplan-Meier method was used to depict survival distribution, and the log-rank test was used for comparison. DCR was defined as the percentage of patients showing complete response (CR), partial response (PR) and stable disease (SD) to treatment based on RECIST 1.1. RR was defined as the percentage of patients who had achieved CR and PR based on RECIST 1.1. PFS was defined as the time from the initiation of therapy until evidence of disease progression or death. OS was defined as the time from the initiation of therapy until death from any cause. A $P$ value $(P)<0.05$ was considered statistically significant.

\section{RESULTS}

\section{Clinical outcomes}

A total of 53 patients were enrolled, of which 7 were excluded due to rapid progression or withdrawal. Patient characteristics included median age 55 years (range, 31-82); male (85\%); ECOG performance status 0/1/2 (11\%/76\%/13\%); locoregional/metastatic/both (30\%/32\%/38\%); oral cavity/oropharynx/larynx primary (36\%/30\%/13\%); prior chemotherapy regimens $1 / \geq 2$ (38\%/62\%; Table 1).

In the buparlisib monotherapy phase, 35 patients were evaluated. DCR was $49 \%$ and RR was 3\% (Fig. 1a). After protocol revision, 11 patients who showed progression under buparlisib monotherapy were treated with the combination of buparlisib and cetuximab. In the combination phase, DCR was $91 \%$ and RR was $18 \%$ (Fig. 1b). Median PFS for buparlisib, followed by combination therapy was significantly longer than that for buparlisib monotherapy (63 and 111 days, $P=0.039$; Fig. 1c). Median OS for buparlisib followed by combination therapy tended to be prolonged compared to that for buparlisib monotherapy (148 and 205 days, $P=0.19$; Fig. 1 d).

Treatment-related AEs are summarised in Supplementary Table S2. Grade 3-4 AEs were reported in 18 of 35 patients in the buparlisib monotherapy group and 4 of 11 patients in the 
Table 1. Baseline patient characteristics.

\begin{tabular}{|c|c|c|}
\hline Characteristic & No. of patients & $\%$ \\
\hline \multicolumn{3}{|l|}{ Sex } \\
\hline Male & 45 & 85 \\
\hline Female & 8 & 15 \\
\hline \multicolumn{3}{|l|}{ Age (years) } \\
\hline Median (range) & 55 & $(31-82)$ \\
\hline \multicolumn{3}{|l|}{ Performance status } \\
\hline 0 & 7 & 11 \\
\hline 1 & 39 & 76 \\
\hline 2 & 7 & 13 \\
\hline \multicolumn{3}{|l|}{ Smoking history } \\
\hline Never smoker & 16 & 37 \\
\hline Smoker & 37 & 63 \\
\hline \multicolumn{3}{|c|}{ Time from initial diagnosis to study entry (month) } \\
\hline Median (range) & 39 & $(5-159)$ \\
\hline \multicolumn{3}{|l|}{ Primary site } \\
\hline Oral cavity & 19 & 36 \\
\hline Oropharynx & 16 & 30 \\
\hline Hypopharynx & 5 & 9 \\
\hline Larynx & 7 & 13 \\
\hline Sinus (nasal, maxillary and ethmoid) & 6 & 11 \\
\hline \multicolumn{3}{|l|}{ Disease status at study entry } \\
\hline Locoregional & 16 & 30 \\
\hline Distant & 17 & 32 \\
\hline Both & 20 & 38 \\
\hline \multicolumn{3}{|l|}{ Number of prior chemotherapy regimens } \\
\hline 1 & 20 & 38 \\
\hline$\geq 2$ & 33 & 62 \\
\hline
\end{tabular}

combination phase. The most common grade 3-4 AEs were hyperglycaemia (10 in the buparlisib monotherapy vs. 3 in combination phase). Treatment discontinuation for AEs occurred in 19 patients in buparlisib and 3 in combination phase. Treatment-related toxicities were not significantly increased by the combination.

Genomic fidelity of PDX tumours in terms of somatic mutations We performed targeted deep sequencing to detect somatic mutations in seven original tumour (F0) and PDX-F2 tumour pairs to determine whether F0 and F2 samples would exhibit identical mutations. The coincidence of most germline mutations indicated that F0 and F2 samples were nearly identical, with Jaccard similarity index scores of $>80 \%$ for most. ${ }^{30}$ Of the F0 germline mutations identified in the F2 specimens, YHIM-02 and YHIM-01 received the highest scores of $94 \%$ and $92 \%$, respectively (Supplementary Fig. S1). The MAF values for F0-common and F2-common mutations exhibited overall concordance $(R=0.89$ and 0.95 for YHIM-01 and YHIM-02, respectively), suggesting that most mutations in the F2 samples corresponded with those in the F0 samples (Supplementary Fig. S1).

Faithful replication of clinical responses to buparlisib and cetuximab in PDX models

We established YHIM-01, -02, -06 and -07 directly from advanced $\mathrm{R} / \mathrm{M}$ SCCHN patients who were treated in this Phase 2 trial. In addition, YHIM-03, -04 and -05 were established from surgically resected or biopsied SCCHN patients (Supplementary Table S3).

Tissue sections from these models were characterised using H\&E staining and p63 immunohistochemistry (IHC; Supplementary
Fig. S2). The histology of PDX tumours (F2) matched well with that of primary tumours (F0) by both methods. Xenografts expressed squamoid features, including keratinisation and intercellular bridges, found in the original tumours. In seven established F3 generation PDXs, we tested buparlisib or cetuximab alone, or in combination.

To evaluate whether preclinical responses in PDX models mimicked the clinical response in patients, we treated YHIM-01, $-02,-06$ and -07 with buparlisib, in parallel with the corresponding R/M SCCHN patients from whom the PDX tumours were derived, in a prospective trial. We observed that preclinical responses to buparlisib in YHIM-01, $-02,-06$ and -07 precisely replicated the clinical responses of their corresponding patients (Fig. 2a-c). All seven established PDX models had a strong resistance to buparlisib monotherapy, displaying rapid tumour progression within a period of 30 days. Furthermore, marked tumour regression was observed in buparlisib/cetuximab combination therapy compared with buparlisib monotherapy in all PDX models. Notably, YHIM-05, -06 and -07 demonstrated strong resistance to each monotherapy, but treatment with buparlisib/ cetuximab showed more prominently and synergistically inhibited the tumour growth than each buparlisib or cetuximab monotherapy [the average TGI (\%) in YHIM-05, 114.92\% of buparlisib/ cetuximab vs. $58.59 \%$ of buparlisib $(P<0.0001)$ and vs. $20.65 \%$ of cetuximab $(P<0.0001)$; in YHIM-06, 98.01\% of buparlisib/cetuximab vs. $42.37 \%$ of buparlisib $(P<0.001)$ and vs. $78.63 \%$ of cetuximab $(P<0.001)$; in YHIM-07, $102.09 \%$ of buparlisib/cetuximab vs. $52.24 \%$ of buparlisib $(P<0.001)$ and vs. $76.72 \%$ of cetuximab $(P<0.05)$ ] (Fig. 2a and Supplementary Table S4). Data from the PDX and clinical trials were comprehensively integrated to identify predictive biomarkers of buparlisib.

Based on the strong synergy observed in the PDX models, we revised the treatment protocol to the combination for cases that progressed under buparlisib monotherapy. After protocol revision, 11 patients were treated with buparlisib/cetuximab. In the combination phase, PR was observed in $18 \%$ and SD was $46 \%$ in patients who had failed to respond to buparlisib (Fig. 1b).

Interestingly, the patient corresponding to YHIM-07 was enrolled and treated with buparlisib/cetuximab after progression under buparlisib monotherapy based on the revised protocol. Indeed, the response of this patient was similar to that of YHIM-07 (Fig. 2d). After one cycle of buparlisib, the neck node showed progression; however, after adding cetuximab, the neck node improved, showing PR (Fig. 2d). Thus, this patient showed strong synergistic inhibition with the combination, recapitulating the responses observed in the corresponding PDX model. Taken together, faithful replication of the clinical efficacy of buparlisib/ cetuximab in PDX highlights the potential of co-clinical trials to inform and predict clinical outcomes.

Analysis of somatic mutation profiles in PDX tumours Given the similarity of drug sensitivities between xenografts and patients, we set out to identify predictive biomarkers of buparlisib and cetuximab using the genetic and transcriptomic profiles of PDX tumours. Somatic mutations in known cancer-related genes based on the Cancer Gene Census ${ }^{29}$ are shown in Fig. 3a and Supplementary Table S5. Recurrent non-silent mutations $(\geq 2)$ in 10 genes (TP53, SETBP1, FAT1, BCL9, CDKN2A, MECOM, TGFBR2, ERBB2, $K A T 6 B$ and NOTCH1) are shown with mutation types (e.g. missense and nonsense). The annotated amino-acid residue changes of these mutations along with those of singletons are listed in Supplementary Table S5. The most common mutations were TP53 mutations (YHIM-01,-02, -03,-06 and -07). Mutations in FAT1, CDKN2A, NOTCH1 and TGFBR2 have been reported to be frequent in SCCHN; all mutations of these loci in our cohort were found to be truncating (either as nonsense or frameshift) and often appeared as a double hit, suggestive of inactivation of both alleles (i.e. YHIM-06 and -07 showed nonsense and frameshift 

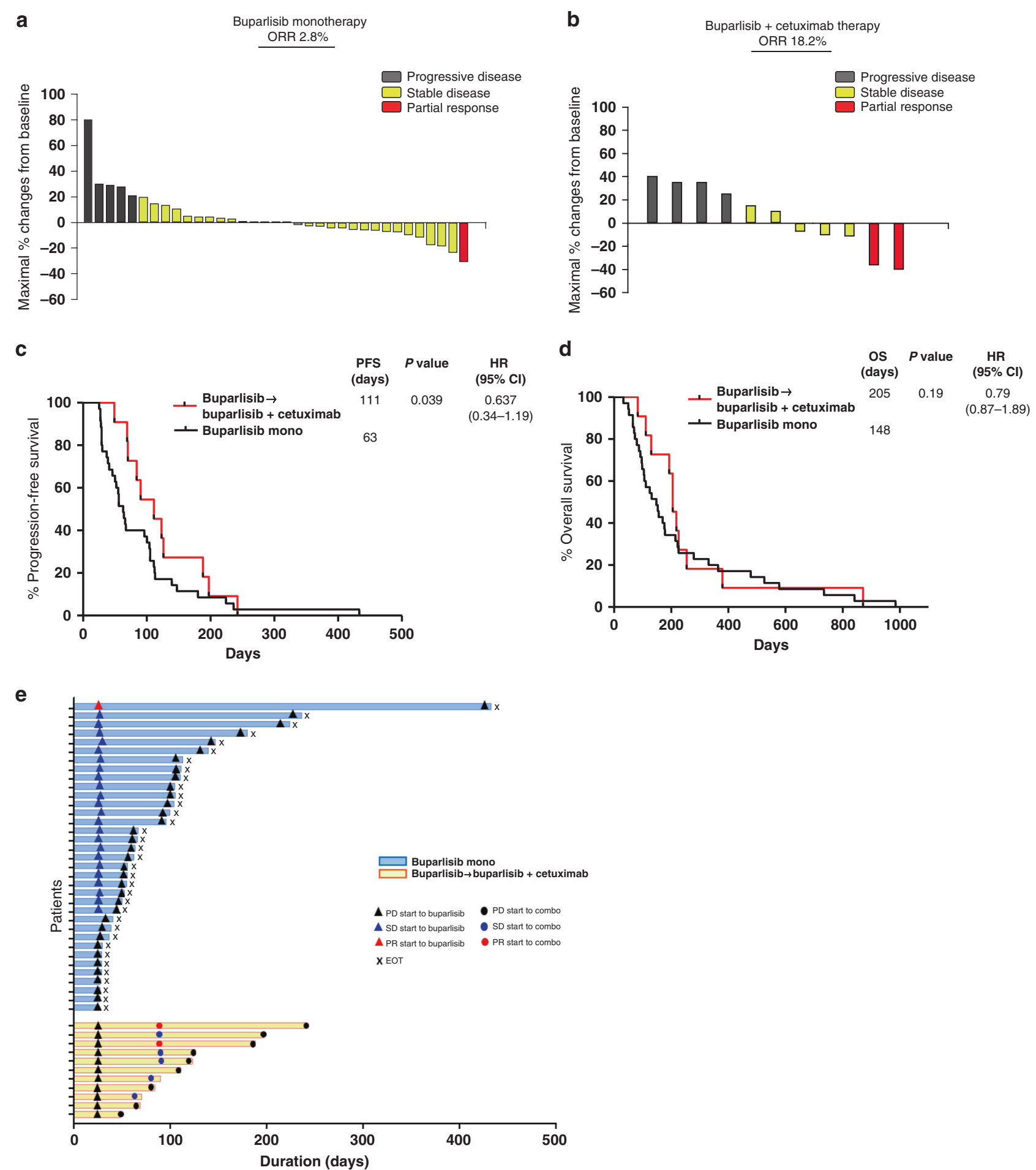

Fig. 1 Clinical response the patients enrolled in clinical trial. a, b Best tumour volume change from baseline in patients with at least one post-baseline measurement a for buparlisib monotherapy, and $\mathbf{b}$ for the buparlisib/cetuximab combination phase. $\mathbf{c}$ Kaplan-Meier curves for progression-free survival. (d) Kaplan-Meier curve for overall survival. (e) Duration of response.

mutations for FAT1 and NOTCH1, respectively). However, no PIK3CA mutations or recently reported novel SCCHN-related mutations, such as AJUBA and NSD1, were found.

We also examined singleton mutations in cancer-related genes. Of note, YHIM-05 harboured mutations in HRAS and CASP8, but retained wild-type TP53, which is expected to have favourable clinical outcomes, and a deficit of SCNAs ${ }^{7}$ was found as a synergy group. For the recurrent cancer-related genes shown in
Supplementary Table S5, no mutations showed significant enrichment toward cases with synergy; this was also true for the remaining non-silent mutations in all the mutated genes. The somatic mutations identified in seven cases are presented in Supplementary Table 6.

The mutation rates (i.e. the number of exonic mutations per $\mathrm{Mb}$ ) for all seven PDX models are shown in Fig. 3a. Cases corresponding to YHIM-05, -06 and -07 showed relatively elevated mutation 
a
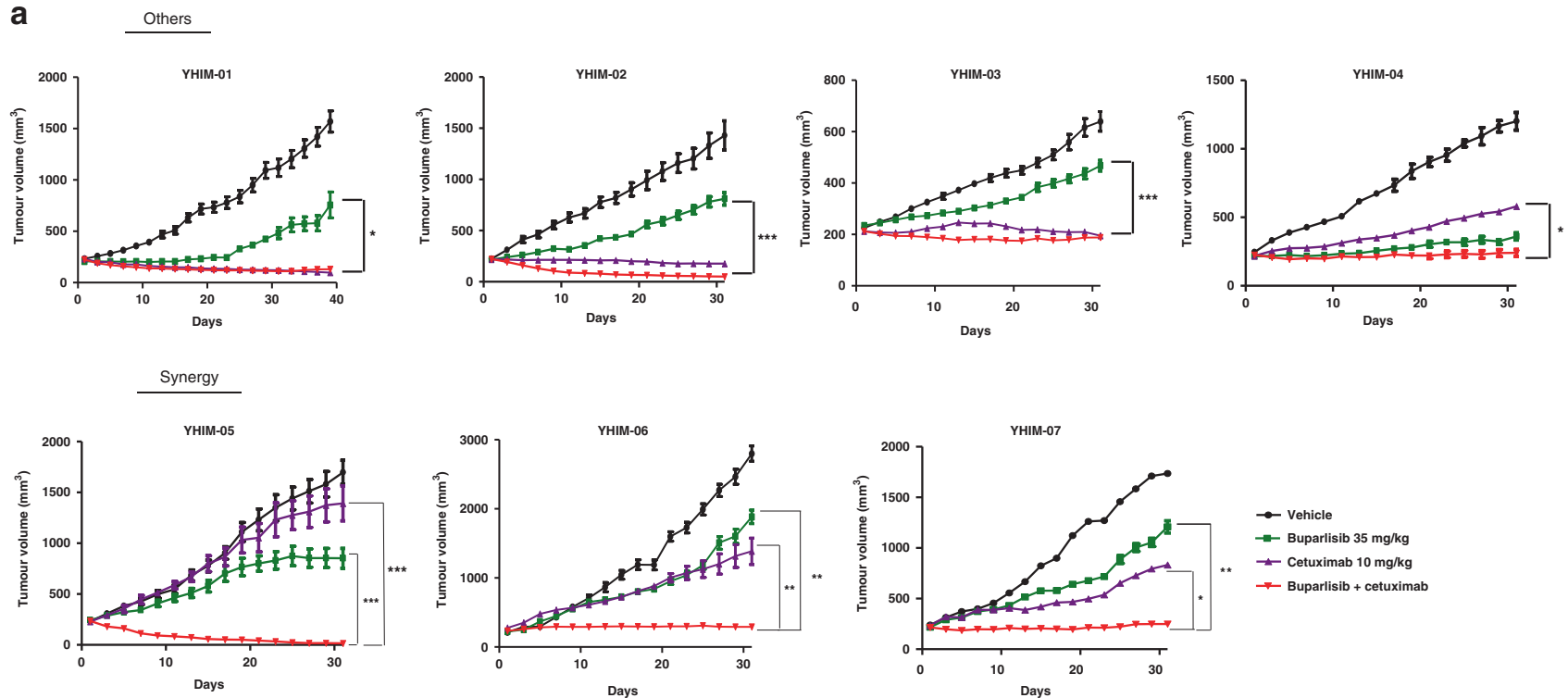

Erbitux $10 \mathrm{mg} / \mathrm{kg}$ vs. BKM120 $35 \mathrm{mg} / \mathrm{kg}+$ erbitux $10 \mathrm{mg} / \mathrm{kg}$ BKM120 $35 \mathrm{mg} / \mathrm{kg}$ vs. BKM120 $35 \mathrm{mg} / \mathrm{kg}+$ erbitux $10 \mathrm{mg} / \mathrm{kg}$

Using one-way ANOVA, ${ }^{*}<0.05$
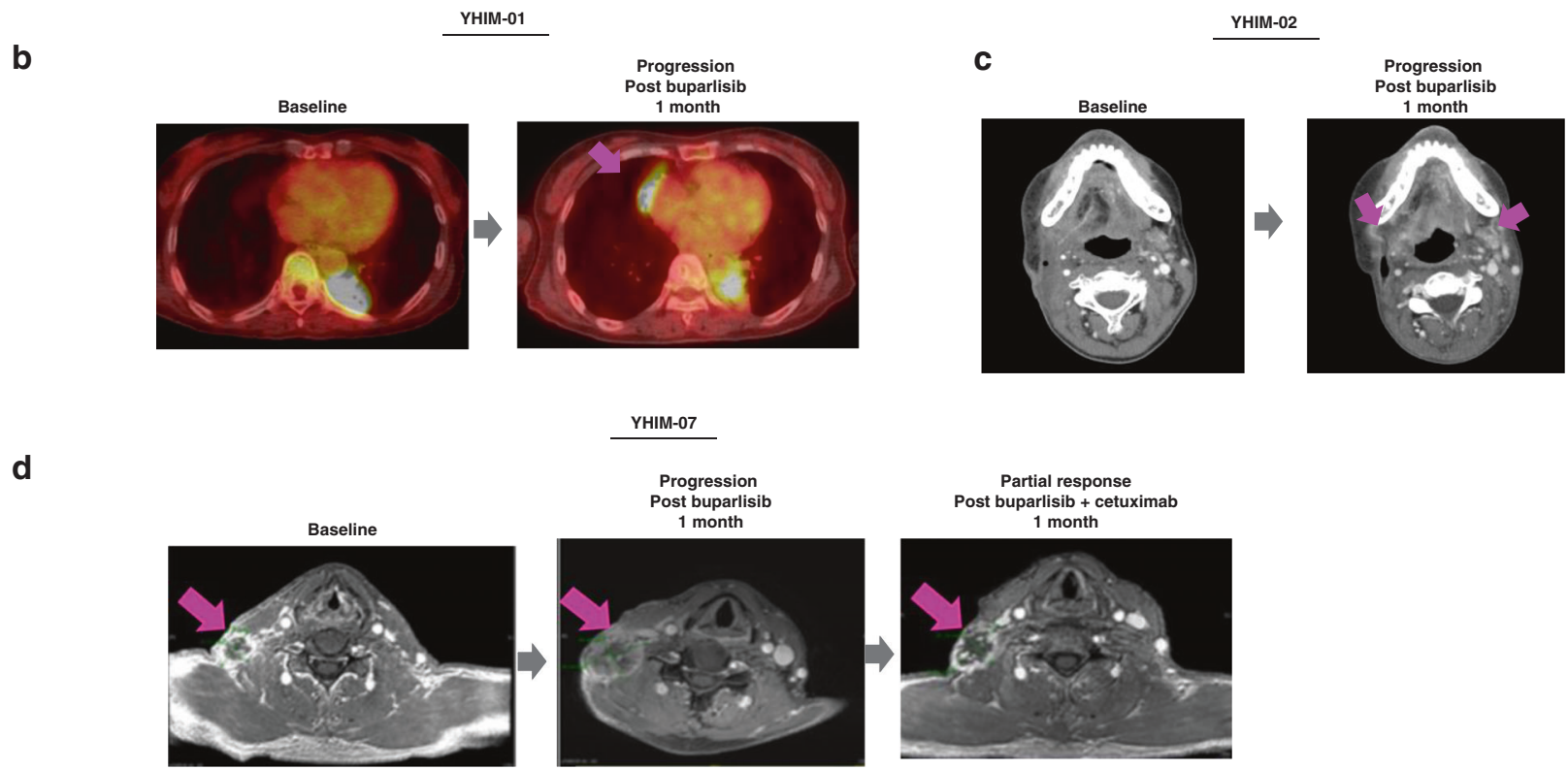

Fig. 2 Drug responses of PDX models and corresponding patients. a Buparlisib, cetuximab and buparlisib/cetuximab tested in PDX models. b Correlation between patient YHIM-01 and PDX treated with buparlisib. c Rapid progression of patient YHIM-02 and PDX under buparlisib monotherapy. d Response of patient YHIM-07 to buparlisib/cetuximab therapy after progression under buparlisib monotherapy. After one cycle of buparlisib, the neck node progressed; however, it improved after the addition of cetuximab. Pink arrowhead indicates the measurable target tumour lesions.

burdens (6.3-7.8 mutations/Mb) compared with the others (YHIM$01,-02,-03$ and -04$)(1.7-6.7$ mutations/Mb) $(P=0.075)$. The relative proportions of missense mutations as well as mutation signatures are illustrated in Fig. 3a. The nonsynonymous/synonymous ratio was relatively low $(<2.0)$ for four cases (YHIM-01, -03 , -05 and -06), suggesting that xenocontamination was still substantial and may have produced elevated mutation rates for those cases (asterisks in Fig. 3a). Mutation signature analysis revealed age-related signatures (signatures $1 \mathrm{~A}$ and $1 \mathrm{~B}$; annotated as previously proposed) ${ }^{31}$ that were universally observed across the genomes examined (17-46\%). Previous signature analyses on TCGA head and neck cancer genomes revealed that APOBEC, smoking and ultraviolet radiation-related signatures (signature 2/
13,4 and 7, respectively) comprise the major mutation signatures of SCCHN. ${ }^{7}$ In this study, these signatures were observed in 4, 2 and 1 case(s), respectively.

We also investigated somatic mutations in 31 patients from whom tumour tissue was available. Significant genetic alterations related to drug response were not observed (Supplementary Fig. S3). Because there were no matched normal samples for these 31 patients, we investigated all the mutations of these samples using HaplotypeCaller. Thereafter, to assess the cancer-specific mutations, we employed $\mathrm{CIVic}^{26}$ and $\mathrm{DoCM}^{27}$ which are specialised databases for interpretation of cancer mutations. Consequently, there were no targetable mutations, such as PIK3CA E542K, E545K and H1047R, which are known as actionable 
a

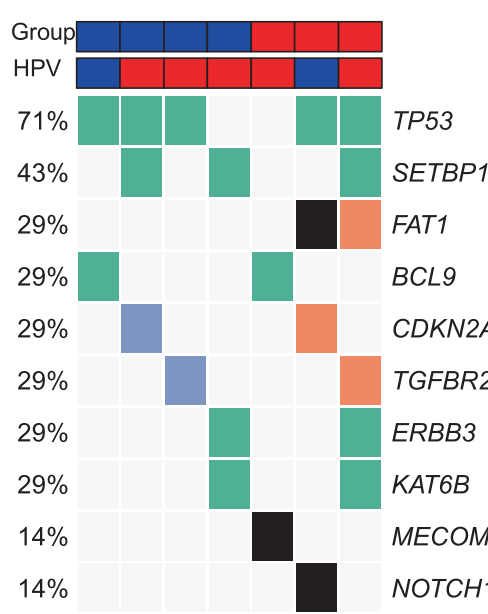

$10-$
$8-1$
$6-$
$4-\square$
$2-\square$

1
0.8
0.6
0.4
0.2
0.0
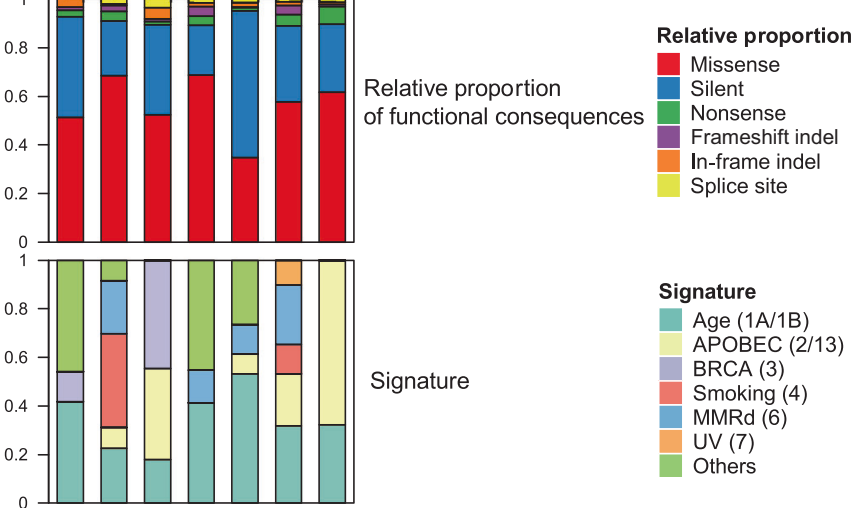

Signature
Group HPV

Others Pos

Synergy $\square$ Neg

Alterations

Missense

Frameshift indel

Multiple
Mutation rate

Splice site

\section{b}

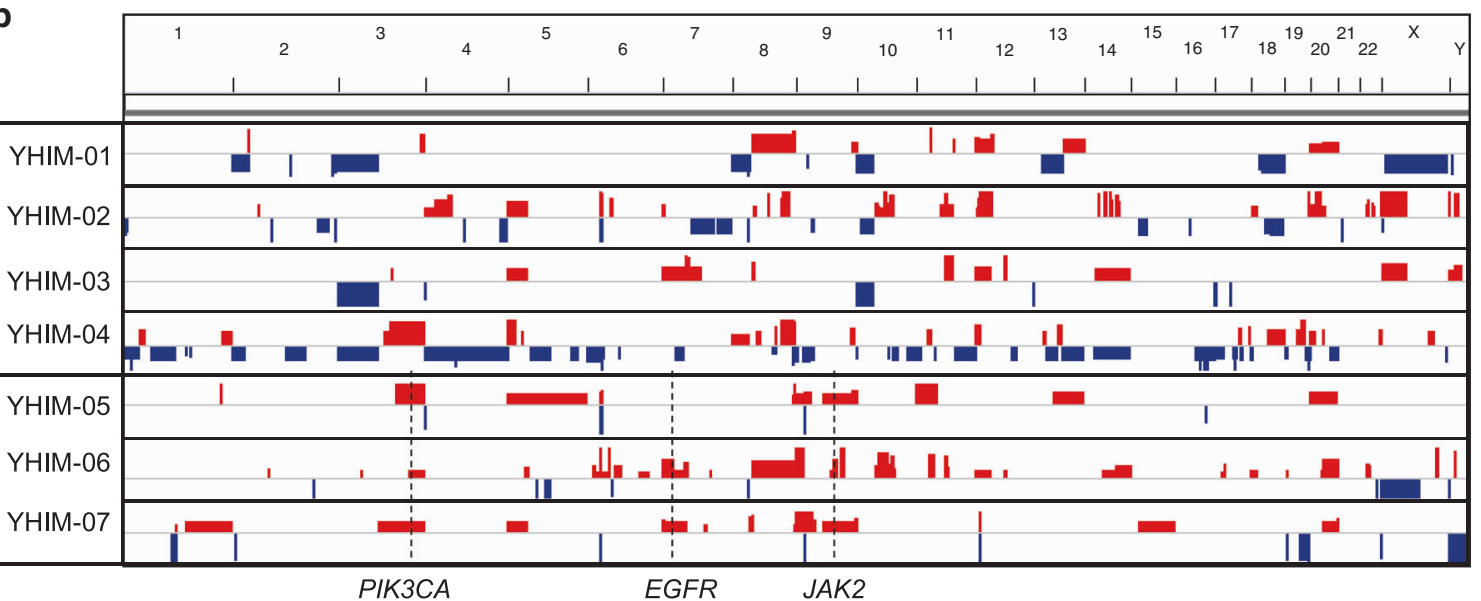

Fig. 3 Genetic alteration profile in patient-derived xenograft models. a Somatic mutations in known cancer-related genes based on the Cancer Gene Census for all seven PDX models (first panel); mutation rates for all seven PDX models (second panel); relative proportions of functional consequences (third panel); and signatures (fourth panel). b Genome-wide somatic copy number alteration profiles of seven PDX models using whole-exome sequencing data. Asterisks indicate the cases with NS/S (nonsynonymous/synonymous) ratios less than 2.0. 
a

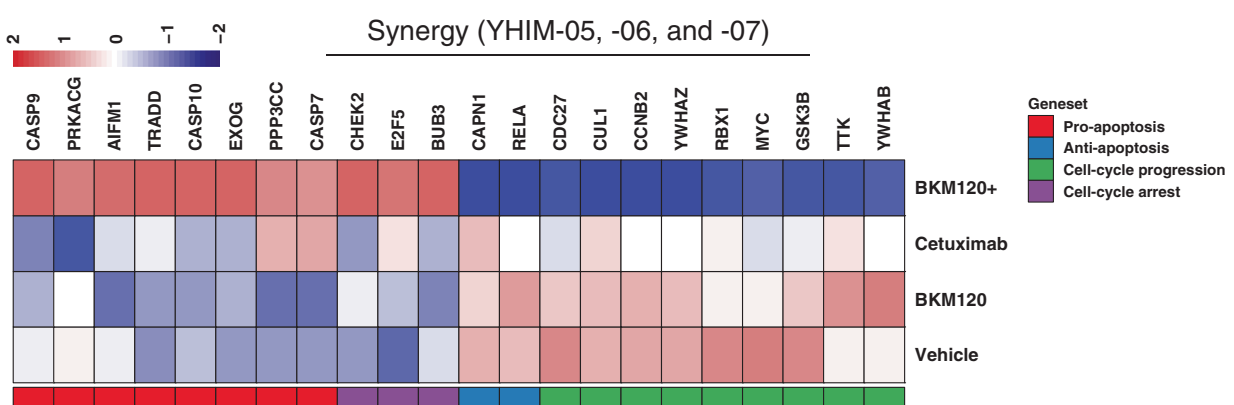

b

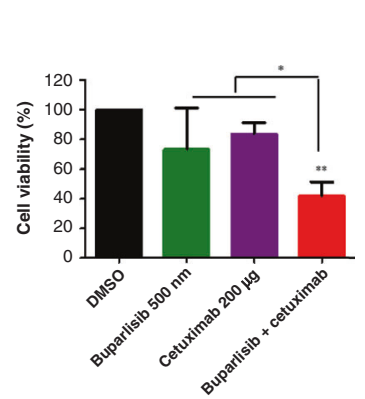

c

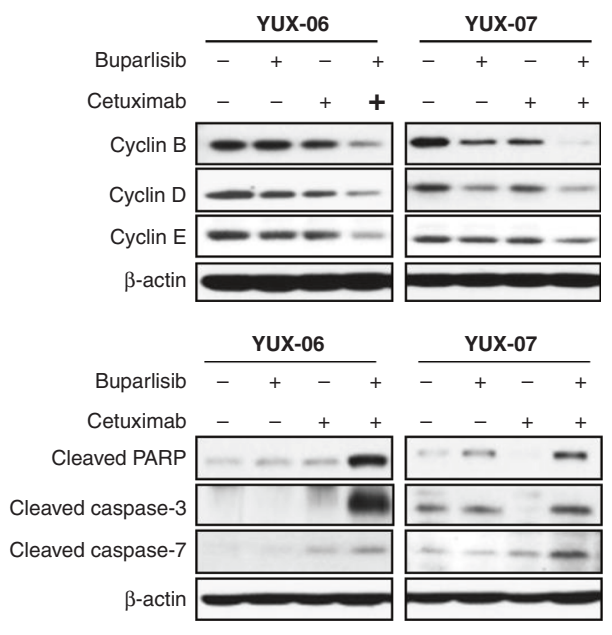

YUX-06
DMSO Buparlisib $\underset{500 \mathrm{nM}}{\substack{\text { Cetuximab Buparlisib + } \\ \text { cetuximab }}}$
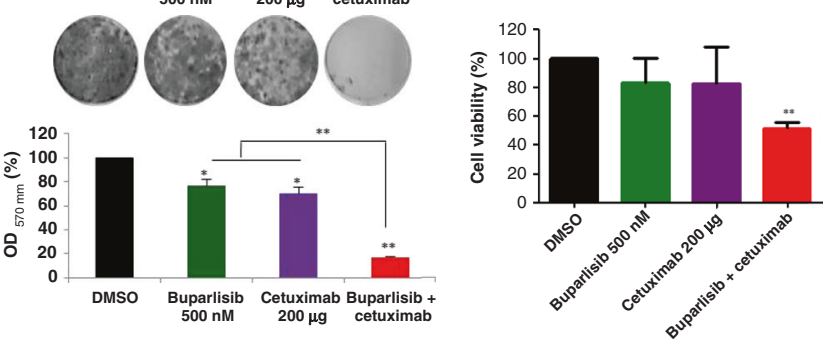

d
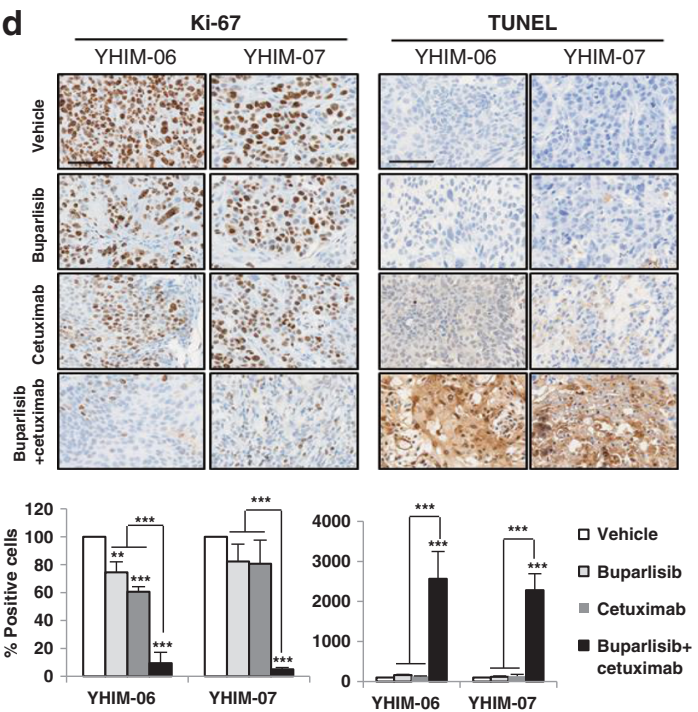

e
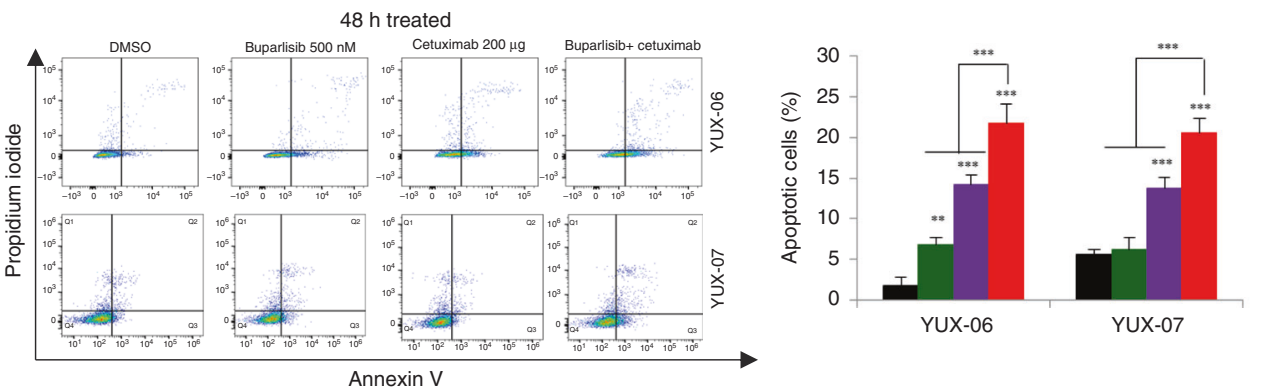

- DMSO

- Buparlisib

- Cetuximab

- Buparlisib

+ cetuximab

Fig. 4 In vitro experiments for cell proliferation in the PDX models showing synergy of buparlisib/cetuximab. a Comparison of gene expression profiles between buparlisib, cetuximab, buparlisib/cetuximab and control in PDX models YHIM- 05 , -06 and -07 , showing that genes associated with apoptosis and cell-cycle arrest were significantly upregulated by combination therapy. $\mathbf{b}$ Cell viability as a function of drug treatment of cell lines from PDX models YUX-06 and -07). c Expression of genes related to cell-cycle progression (cyclins B, D and E) and apoptosis (cleaved PARP, cleaved caspase-3 and cleaved-7) in YUX-06 and -07. d IHC showing proliferative (Ki67) and apoptotic (TUNEL) markers in YHIM-06 and -07. e Flow cytometry showing apoptosis in YUX-06 and -07. 
mutations. Supplementary Figure S3A was drawn to confirm the pattern of the cancer-specific mutations. TP53 (1 for L72Q, 1 for Y166C, 1 for Y181C, 1 for R209W, 2 for R234H), MLH1 (3 for V384D), CDKN2A (1 for R80X, 1 for W110X), STK11 (1 for P281L) and MAPK1 (1 for E332K) mutations were included in the CIVic and DoCM databases. Supplementary Figure S3B is a needle plot showing the hotspot mutations of TP53.

Copy number alteration profiles in PDX tumours

SCNAs were evaluated using WES data. Genome-wide SCNA profiles of seven cases examined are illustrated in Fig. $3 \mathrm{~b}$. Recurrent losses were observed in $3 p$ and $10 p$, along with recurrent gains in $3 q, 5 p, 8 q, 9 p / q, 12 q$ and $20 p / q$, consistent with the previously reported frequencies of SCNA in SCCHN genomes. ${ }^{7}$ We also examined focal, gene-level amplifications or deletions of genes previously reported to be related to $\mathrm{SCCHN}^{7}$ including the locus chr9:1-17 Mb (Fig. 3b). Of note, the amplification of PIK3CA and JAK2 was relatively frequent for YHIM-05, -06 and -07 , and EGFR amplification was observed in YHIM-06 and -07 (Fig. 3b).

Combination treatment upregulated genes associated with apoptotic and cell-cycle arrest

To elucidate the mechanism underlying the synergistic effect of buparlisib and cetuximab, we performed a comparative analysis of gene expression in YHIM-05, -06 and -07 at baseline, after buparlisib or cetuximab alone and after combination therapy. Genes associated with apoptotic and cell-cycle arrest were significantly upregulated with combination therapy compared with that after each buparlisib or cetuximab monotherapy. In contrast, the expression of genes related to anti-apoptotic and cell-cycle progression was downregulated (Fig. 4a).

To explore the mechanism of combination therapy, we performed cell viability assays and immunoblots using PDXderived tumour cell lines from YUX-06 and -07 (YHIM-06 and -07). The combination of buparlisib with cetuximab induced a synergistic anti-tumour effect, as evidenced by $\mathrm{Cl}<1(\mathrm{Cl}$ value, 0.628 (YUX-06); 0.756 (YUX-07)) and induced apoptosis (Fig. 4b). Dual inhibition of the EGFR and PI3K pathways induced the downregulation of molecules related to cell-cycle progression (cyclin B, cyclin D and cyclin E) and upregulation of molecules related to apoptosis (cleaved PARP, cleaved caspase-3 and caspase-7) to greater extents than single inhibition (Fig. 4c). The combination treatment significantly increased apoptosis and decreased proliferation in YHIM-06 and -07 based on IHC $(P<$ 0.001 ; Fig. 4d). Moreover, apoptosis was dramatically increased in YUX-06 and -07 by flow cytometry $(P<0.001$; Fig. 4e). In YUX-01 and -02 , the levels of $\mathrm{BCl}-2$, cleaved caspase- 9 and Myc were not changed by buparlisib monotherapy; however, increased cleaved caspase- 9 and decreased $\mathrm{Bcl}-2$ and Myc were observed after cetuximab as well as after buparlisib/cetuximab combination therapy (Supplementary Figure S4).

\section{DISCUSSION}

This study showed that the pan-PI3K inhibitor buparlisib was insufficient for the treatment of patients with R/M SCCHN, even though $\mathrm{PI} 3 \mathrm{~K}$ pathway alterations are frequently caused by mutations associated with SCCHN.,8 We conducted a mouse-human co-clinical trial, with PDX models derived from biopsied tumour samples of the patients enrolled in this Phase 2 clinical trial. This co-clinical trial indicated that all PDX models were resistant to buparlisib monotherapy, while some PDX models showed remarkable sensitivity to the combination of buparlisib/ cetuximab. Based on these results, we revised the clinical trial protocol and switched patients who failed to respond to buparlisib monotherapy to the combination of buparlisib/cetuximab. The combination improved treatment outcomes without any significant increase in treatment-related toxicities.
Transcriptomic analyses indicated that genes related to apoptosis and cell-cycle arrest were significantly upregulated upon combination treatment compared with treatment with buparlisib or cetuximab alone.

We successfully established PDX models from R/M SCCHN patients and conducted a co-clinical trial in parallel with a Phase 2 clinical trial. We demonstrated that histologic and genetic characteristics were highly preserved between patient tumours and corresponding PDX tumours. Although the PI3K-AKT-mTOR pathway is frequently activated in SCCHN, ${ }^{7,8}$ the efficacy data from the clinical trial as well as from PDX models showed the tumour to be highly resistant to buparlisib monotherapy. Our findings indicated that the combination treatment induced a synergistic anti-tumour effect as evidenced by $\mathrm{Cl}<1$, and induced apoptosis and cell-cycle arrest. To exclude exactly that combination regimen is not the effect of cetuximab monotherapy, the clinical study design comparing combination (buparlisib/cetuximab), buparlisib monotherapy and cetuximab monotherapy is necessary. There were lots of clinical trials with cetuximab monotherapy in head and neck cancer patients. In general, the RR of cetuximab monotherapy has been known as $10-20 \%{ }^{32}$ even though the RR was different depending on the trials.

To identify predictive genomic alterations in response to combination therapy, we conducted comprehensive genomic and transcriptomic analyses of the baseline and on-treatment tumour samples. Unexpectedly, predictive genomic alterations upon combination therapy were not observed in either PDX or patient samples, which would be limited by a small sample size. Expression of genes associated with apoptosis and cell-cycle arrest was significantly upregulated by combination treatment. These preclinical data strongly suggest that combination therapies with buparlisib and cetuximab exert their effects by facilitating apoptosis and cell-cycle arrest. Thus, the therapeutic strategy of PI3K inhibitor plus EGFR monoclonal antibody could improve treatment outcomes in R/M SCCHN patients.

Treatment-related toxicities must be considered when developing combination therapies for cancer patients. Known AEs associated with buparlisib, including hyperglycaemia and gastrointestinal AEs (e.g. stomatitis, diarrhoea, nausea and vomiting), could be managed with the established strategies of dose reduction and treatment of symptoms with appropriate concomitant medication. Moreover, the occurrence of AEs related to cetuximab, including skin rash, mucositis or diarrhoea, ${ }^{3}$ was not increased in patients by the combination therapy. However, in the combination phase of this study, patients are congruent with the $\mathrm{AE}$ of these combined agents and high discontinuation rate. This study has a limitation that only 11 patients were treated with buparlisib/cetuximab after revising the protocol, which is a small number. Thus, cautious interpretation and additional clinical trials are needed to confirm our results.

To our knowledge, this is the first study demonstrating the additive or synergistic effects of buparlisib and cetuximab in a clinical trial based on preclinical PDX data, which can significantly contribute to the clinical development of PI3K inhibitors in R/M SCCHN patients. A combination of buparlisib/cetuximab may overcome resistance to buparlisib and represent a more effective option for treating patients with R/M SCCHN.

\section{ACKNOWLEDGEMENTS}

We thank the patients and their families who participated in this clinical trial, and coinvestigators and clinical research coordinators for processing this clinical trial.

\section{AUTHOR CONTRIBUTIONS}

Study concepts: H.R.K., T.-M.K. and B.C.C. Study design: H.R.K., H.N.K., T.-M.K. and B.C.C. Data acquisition: H.R.K., H.N.K., K.Y.J., J.W.C., D.M.J., K.H.P., M.H.H., M.-J.A., J.-M.S. and H.S.K. Quality control of data and algorithms: J.K., J.Y., K.R.K., Y.W.K., S.H.K., E.C.C., S.O.Y., H.S.S. and S.P. Data analysis and interpretation: H.R.K., H.N.K., T.-M.K. and BCC. Statistical 
analysis: J.K., J.Y., J.W.C. and D.M.J. Manuscript preparation: H.R.K., H.N.K., T.-M.K. and B.C. C. Manuscript editing: H.R.K., H.N.K., T.-M.K. and B.C.C. Manuscript review: All authors reviewed the manuscript.

\section{ADDITIONAL INFORMATION}

Ethics approval and consent to participate This study was conducted under approval by the institutional review boards of Severance Hospital and in accord with the 1964 Declaration of Helsinki and its later amendments or comparable ethical standards. All patients provided informed consent. All animal studies were performed in accordance with the guidelines of the Animal Research Committee of Yonsei University College of Medicine and were approved by the Association of Assessment and Accreditation of Laboratory Animal Care.

Data availability All data presented within the article and its Supplementary information files are available upon request from the corresponding author.

Competing interests The authors declare no competing interests.

Funding information This work was supported by a grant from the Korea Health Technology R\&D Project through the Korea Health Industry Development Institute (KHIDI), Basic Science Research Programme through the NRF funded by the Ministry of Science and ICT (2016R1A2B3016282 to B.C.C.) and the Korea Health Technology R\&D Project through the Korea Health Industry Development Institute (KHIDI), funded by the Ministry of Health \&Welfare, Republic of Korea (HI13C2162 to S.P.).

Supplementary information is available for this paper at https://doi.org/10.1038/ s41416-020-01074-2.

Publisher's note Springer Nature remains neutral with regard to jurisdictional claims in published maps and institutional affiliations.

\section{REFERENCES}

1. Hunter, K. D., Parkinson, E. K. \& Harrison, P. R. Profiling early head and neck cancer. Nat. Rev. Cancer 5, 127-135 (2005).

2. Lim, S. M., Cho, S. H., Hwang, I. G., Choi, J. W., Chang, H., Ahn, M. J. et al. Investigating the feasibility of targeted next-generation sequencing to guide the treatment of head and neck squamous cell carcinoma. Cancer Res. Treat. 51, 300-312 (2019).

3. Vermorken, J. B., Trigo, J., Hitt, R., Koralewski, P., Diaz-Rubio, E., Rolland, F. et al. Open-label, uncontrolled, multicenter phase II study to evaluate the efficacy and toxicity of cetuximab as a single agent in patients with recurrent and/or metastatic squamous cell carcinoma of the head and neck who failed to respond to platinum-based therapy. J. Clin. Oncol. 25, 2171-2177 (2007).

4. Cohen, E. E. W., Soulieres, D., Le Tourneau, C., Dinis, J., Licitra, L., Ahn, M. J. et al. Pembrolizumab versus methotrexate, docetaxel, or cetuximab for recurrent or metastatic head-and-neck squamous cell carcinoma (KEYNOTE-040): a randomised, open-label, phase 3 study. Lancet 393, 156-167 (2019).

5. Ferris, R. L., Blumenschein, G. Jr., Fayette, J., Guigay, J., Colevas, A. D., Licitra, L. et al. Nivolumab for recurrent squamous-cell carcinoma of the head and neck. $N$. Engl. J. Med. 375, 1856-1867 (2016).

6. Bauml, J., Seiwert, T. Y., Pfister, D. G., Worden, F., Liu, S. V., Gilbert, J. et al. Pembrolizumab for platinum- and cetuximab-refractory head and neck cancer: results from a single-arm, phase II study. J. Clin. Oncol. 35, 1542-1549 (2017).

7. Cancer Genome Atlas, N. Comprehensive genomic characterization of head and neck squamous cell carcinomas. Nature 517, 576-582 (2015).

8. Lui, V. W., Hedberg, M. L., Li, H., Vangara, B. S., Pendleton, K., Zeng, Y. et al. Frequent mutation of the PI3K pathway in head and neck cancer defines predictive biomarkers. Cancer Discov. 3, 761-769 (2013).

9. Soulieres, D., Faivre, S., Mesia, R., Remenar, E., Li, S. H., Karpenko, A. et al. Buparlisib and paclitaxel in patients with platinum-pretreated recurrent or metastatic squamous cell carcinoma of the head and neck (BERIL-1): a randomised, doubleblind, placebo-controlled phase 2 trial. Lancet Oncol. 18, 323-335 (2017).

10. Hidalgo, M., Amant, F., Biankin, A. V., Budinska, E., Byrne, A. T., Caldas, C. et al. Patient-derived xenograft models: an emerging platform for translational cancer research. Cancer Discov. 4, 998-1013 (2014).

11. Shoemaker, R. H. The NCI60 human tumour cell line anticancer drug screen. Nat. Rev. Cancer 6, 813-823 (2006).

12. Abaan, O. D., Polley, E. C., Davis, S. R., Zhu, Y. J., Bilke, S., Walker, R. L. et al. The exomes of the $\mathrm{NCl}-60$ panel: a genomic resource for cancer biology and systems pharmacology. Cancer Res. 73, 4372-4382 (2013).
13. Ilie, M., Nunes, M., Blot, L., Hofman, V., Long-Mira, E., Butori, C. et al. Setting up a wide panel of patient-derived tumor xenografts of non-small cell lung cancer by improving the preanalytical steps. Cancer Med. 4, 201-211 (2015).

14. Fritsch, M., Schmidt, N., Groticke, I., Frisk, A. L., Keator, C. S., Koch, M. et al. Application of a patient derived xenograft model for predicative study of uterine fibroid disease. PLOS ONE 10, e0142429 (2015).

15. Kim, H. R., Kang, H. N., Shim, H. S., Kim, E. Y., Kim, J., Kim, D. J. et al. Co-clinical trials demonstrate predictive biomarkers for dovitinib, an FGFR inhibitor, in lung squamous cell carcinoma. Ann. Oncol. 28, 1250-1259 (2017).

16. Eisenhauer, E. A., Therasse, P., Bogaerts, J., Schwartz, L. H., Sargent, D., Ford, R. et al. New response evaluation criteria in solid tumours: revised RECIST guideline (version 1.1). Eur. J. Cancer 45, 228-247 (2009).

17. Bijnsdorp, I. V., Giovannetti, E. \& Peters, G. J. Analysis of drug interactions. Methods Mol. Biol. 731, 421-434 (2011).

18. Bray, F., Ferlay, J., Soerjomataram, I., Siegel, R. L., Torre, L. A. \& Jemal, A. Global cancer statistics 2018: GLOBOCAN estimates of incidence and mortality worldwide for 36 cancers in 185 countries. CA Cancer J. Clin. 68, 394-424 (2018).

19. Conway, T., Wazny, J., Bromage, A., Tymms, M., Sooraj, D., Williams, E. D. et al. Xenome- - a tool for classifying reads from xenograft samples. Bioinformatics 28 i172-i178 (2012).

20. Li, H. \& Durbin, R. Fast and accurate long-read alignment with Burrows-Wheeler transform. Bioinformatics 26, 589-595 (2010).

21. McKenna, A., Hanna, M., Banks, E., Sivachenko, A., Cibulskis, K., Kernytsky, A. et al. The Genome Analysis Toolkit: a MapReduce framework for analyzing nextgeneration DNA sequencing data. Genome Res. 20, 1297-1303 (2010).

22. do Valle, I. F., Giampieri, E., Simonetti, G., Padella, A., Manfrini, M., Ferrari, A. et al. Optimized pipeline of MuTect and GATK tools to improve the detection of somatic single nucleotide polymorphisms in whole-exome sequencing data. BMC Bioinform. 17, 341 (2016).

23. Xu, D., Olman, V., Wang, L. \& Xu, Y. EXCAVATOR: a computer program for efficiently mining gene expression data. Nucleic Acids Res. 31, 5582-5589 (2003).

24. Tso, K. Y., Lee, S. D., Lo, K. W. \& Yip, K. Y. Are special read alignment strategies necessary and cost-effective when handling sequencing reads from patientderived tumor xenografts? BMC Genomics 15, 1172 (2014).

25. Wang, K., Li, M. \& Hakonarson, H. ANNOVAR: functional annotation of genetic variants from high-throughput sequencing data. Nucleic Acids Res. 38, e164 (2010).

26. Griffith, M., Spies, N. C., Krysiak, K., McMichael, J. F., Coffman, A. C., Danos, A. M. et al. CIViC is a community knowledgebase for expert crowdsourcing the clinical interpretation of variants in cancer. Nat. Genet. 49, 170-174 (2017).

27. Ainscough, B. J., Griffith, M., Coffman, A. C., Wagner, A. H., Kunisaki, J., Choudhary, M. N. et al. DoCM: a database of curated mutations in cancer. Nat. Methods 13 806-807 (2016).

28. Trapnell, C., Roberts, A., Goff, L., Pertea, G., Kim, D., Kelley, D. R. et al. Differential gene and transcript expression analysis of RNA-seq experiments with TopHat and Cufflinks. Nat. Protoc. 7, 562-578 (2012).

29. Gu, Z., Eils, R. \& Schlesner, M. Complex heatmaps reveal patterns and correlations in multidimensional genomic data. Bioinformatics 32, 2847-2849 (2016).

30. Mayakonda, A., Lin, D. C., Assenov, Y., Plass, C. \& Koeffler, H. P. Maftools: efficient and comprehensive analysis of somatic variants in cancer. Genome Res. 28 1747-1756 (2018).

31. Alexandrov, L. B., Nik-Zainal, S., Wedge, D. C., Aparicio, S. A., Behjati, S., Biankin, A. V. et al. Signatures of mutational processes in human cancer. Nature 500, 415-421 (2013).

32. Fury, M. G., Sherman, E., Lisa, D., Agarwal, N., Algazy, K., Brockstein, B. et al. A randomized phase II study of cetuximab every 2 weeks at either 500 or $750 \mathrm{mg} /$ $\mathrm{m} 2$ for patients with recurrent or metastatic head and neck squamous cell cancer. J. Natl Compr. Canc. Netw. 10, 1391-1398 (2012).

Open Access This article is licensed under a Creative Commons Attribution 4.0 International License, which permits use, sharing, adaptation, distribution and reproduction in any medium or format, as long as you give appropriate credit to the original author(s) and the source, provide a link to the Creative Commons license, and indicate if changes were made. The images or other third party material in this article are included in the article's Creative Commons license, unless indicated otherwise in a credit line to the material. If material is not included in the article's Creative Commons license and your intended use is not permitted by statutory regulation or exceeds the permitted use, you will need to obtain permission directly from the copyright holder. To view a copy of this license, visit http://creativecommons.org/licenses/ by/4.0/.

(c) The Author(s) 2020 